\title{
CHRONIC MULTIPLE KNEE LIGAMENT INJURIES: EPIDEMIOLOGICAL ANALYSIS OF MORE THAN ONE HUNDRED CASES
}

\author{
Rosalvo Zosimo Bispo Júnior, ${ }^{a}$ Cezar Teruyuki Kawano, ${ }^{b}$ Alexandre Vieira \\ Guedes $^{b}$
}

Bispo Júnior, RZ, Kawano CT, Guedes AV. Chronic multiple knee ligament injuries: epidemiological analysis of more than one hundred cases. Clinics. 2008;63(1):3-8.

INTRODUCTION: Diagnosis and treatment of multiple ligament injuries of the knee remain a real challenge for most surgeons. OBJECTIVE: To find out the epidemiological profile of patients surgically treated at a Reference Service with more than one chronic ligament injury in the knee joint.

MATERIALS AND METHODS: Of a total of 978 operated patients, 109 presented at least two associated ligament injuries in the same knee. Demographic and clinical variables were evaluated.

RESULTS: The anterior cruciate ligament group presented a larger number of cases of ligament injuries related with sports practice and falls, while the posterior cruciate ligament and anterior cruciate ligament + posterior cruciate ligament groups presented more cases related to traffic accidents and trauma with object (weight on the knee) $(\mathrm{p}<0.001)$. The varus group presented significantly higher values of time since injury $(\mathrm{p}<0.01)$. In the group with new anterior cruciate ligament injury (neoligament) associated with other ligament injuries the disruption times were higher, showing statistical significance $(\mathrm{p}<0.001)$.

CONCLUSIONS: Anterior cruciate ligament injury associated with other ligament injuries other than posterior cruciate ligament injury are related to sports practice and falls. Posterior cruciate ligament injury associated to other ligament injuries, including or not anterior cruciate ligament injury, are related to traffic accidents and direct trauma caused by an object on the knee. Significant delay between primary ligament injuries and their reconstructions generates varus deformity of the affected knee. In spite of the large delay in seeking medical treatment, few patients with neoligament anterior cruciate ligament injury and other combined disruptions will develop varus deformity.

KEYWORDS: Multiple ligament knee injury. Epidemiological analysis. Surgical treatment. Posteromedial knee injury. Posterolateral knee injury.

\section{INTRODUCTION}

The ligaments of the knee are the primary static stabilizers of the joint and can be divided into the cruciate ligaments and structures of the lateral and medial sides. The cruciate ligaments, consisting of the anterior cruciate ligament (ACL) and the posterior cruciate ligament (PCL), are the most commonly involved in a multiple ligament knee injury. ${ }^{1}$

${ }^{a}$ Biological Science and Health Department, Centro Universitário de João Pessoa - João Pessoa/PB, Brazil.

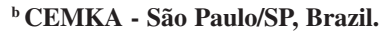

zosimo_jr@yahoo.com.br

Received for publication on August 13, 2007.

Accepted for publication on September 12, 2007.
Multiple ligament injuries of the knee basically concern ruptures of the cruciate ligaments combined with damage to the collateral ligaments. ${ }^{2}$ As such, it is a complex problem in orthopedic surgery. ${ }^{3}$

Diagnosis and treatment of multiple ligament injuries of the knee remain real challenges for most surgeons. These injuries involve the rupture of at least two of the main ligaments of the knee, and are associated with meniscus ruptures, cartilage injuries and osseous fractures. ${ }^{4}$ Surgical treatment may include ligamentous reconstruction, osteotomy, articular cartilage resurfacing and meniscus surgery. ${ }^{5}$

In a knee that is chronically affected by combined ligament injuries, the main objective is to restore biomechani- 
cal stability so that the patient can return to the same level of activity as before the injury. However, it is very difficult to reach this desired level because the injuries are very complex. ${ }^{6}$

Recognition of knee instability has acquired great importance, since a lack of diagnosis may induce severe sequelae, especially when several of these injuries are associated.

The objective of this report is to perform a systematic review, with retrospective analysis of data, of proven cases with more than one chronic ligament injury of the knee joint in patients that were surgically treated in a Reference Service, with the aim of getting to know their epidemiological profile.

\section{SUBJECTS AND METHODS}

\section{Population}

Medical records of patients with a uniform criteria diagnosis of ligament injury of the knee who were surgically treated at a knee pathology Reference Service between October 2003 and December 2006 were reviewed. The preoperative evaluation of the patients included history and physical examination, standard radiographs and magnetic resonance imaging (MRI). A total of 978 patients were identified. Of these, 109 presenting at least two associated ligament injuries in the same knee were eligible for the study. All injuries were chronic (over three weeks in duration ${ }^{2}$ ).

\section{Outlines}

A retrospective analysis of data from proven cases with more than one chronic injury in knee joint ligaments was performed with the aim of determining the epidemiological profile of the patients. Their medical records were examined and the data were recorded in a form that was previously created for this purpose. This protocol was approved by the Ethics Committees of the reference center.

\section{Variables}

Using a standardized protocol applicable to the medical records, we evaluated demographic variables (age, gender and affected half of the body) and clinical variables (causal agent, diagnosis, time since injury, varus deformity and neoligament injury).

\section{Evaluated variables}

Demographic and clinical variables:
- Gender

- Age (years)

- Left and right side of the body

- Causal agent - type of accident that generated the injury, classified into four large groups:

$>$ Sports injuries:

Soccer; Martial Arts

$>$ Traffic injuries:

Motorcycle; Runover; Car accident

$>$ Injuries caused by falls:

Stairs; Floor level; High places

$>$ Injuries caused by weight:

Object on the knee (direct trauma)

- Time since injury - time (in months) elapsed between the accident and specialized medical treatment.

- Varus deformity - presence or absence, acquired after untreated chronic ligament injuries.

- Neoligament injury - presence or absence of lesions to previously reconstructed ligaments associated with other ligamentous disruption(s) of the affected knee.

- Diagnosis - Ligament disruptions were divided into three large groups with their multiple associated injuries: the ACL (Anterior Cruciate Ligament) Group, the PCL (Posterior Cruciate Ligament) Group and the ACL + PCL Group:

\section{ACL Group:}

ACL injury + PLC (Posterolateral Corner) injuries

$A C L$ injury + CLL (Collateral Lateral Ligament) injury

$A C L$ injury + CML (Collateral Medial Ligament) injury

$>$ PCL Group:

$P C L$ injury $+P L C$ injuries

$P C L$ injury + CML injury

ACL + PCL Group:

$A C L$ injury $+P C L$ injury $+P L C$ injuries

\section{Statistical Analysis}

Initially, all variables were descriptively analyzed. For quantitative variables, this analysis was performed by observing the minimum and maximum values and calculating the averages, standards deviations and median values. For qualitative variables, absolute and relative frequencies were calculated.

An analysis of the null hypothesis of means among the three groups was performed by applying one-factor Analysis of Variance (ANOVA) ${ }^{7}$. When the assumption of data normality was rejected, we used the Kruskal-Wallis nonparametric test ${ }^{7}$.

For comparison between two groups, we used the Mann-Whitney ${ }^{7}$ non-parametric test, since the assumption of data normality was rejected. 
We used the chi-square test or Fisher's exact test ${ }^{7}$ (which is employed when less than five frequencies are expected) to test for homogeneity between the percentages of two groups.

The database was prepared using Excel 2002 for Windows following the application of a protocol to the medical records, while the statistical analyses were performed using SPSS (Statistical Package for Social Sciences, version 12.0) statistical software. Statistical significance was defined for $\mathbf{p}$ values $<0.05$.

\section{RESULTS}

Approximately $11 \%$ of all cases of knee ligament injuries operated on in our Service were affected by more than one injury to those structures. Of these, the male gender exceeded females, with $98.2 \%$ of cases.

The age of the patients ranged between 16 and 55 years (mean age: $31.29 \pm 7.8$ years). In most cases $(n=62)$, the right side of the body was the most affected.

The time since injury (time elapsed between the injury and presentation to specialized medical care) ranged between three and 180 months (average: $29.6 \pm 28.1$ months; median value: 24 months).

Sports injuries were considered to be the most frequent causes of multiple ligament injuries of the knee, with 57 $(52.3 \%)$ cases, followed by those caused by traffic accidents, with 31 (28.4\%) cases.

The ACL group, with its associated injuries, presented the highest number of ligament injuries $(n=69)$, followed by the PCL group $(n=23)$ and the ACL + PCL group $(\mathrm{n}=17)$. Among the associated primary injuries, ACL + PLC injuries were the most frequent, with 45 cases.

Respectively, $6.4 \%$ and $8.2 \%$ of patients with more than one knee ligament injury presented varus deformity and neoligament injury of the affected joint.

In addition, the results of some important demographic and clinical variables were evaluated as follows:

\section{DIAGNOSIS GROUPS}

The values for each category of the demographic and clinical variables that were analyzed, as well as the p values, are shown in Table 1. The factor that significantly influenced the diagnosis groups was the type of accident, with the ACL group presenting a larger number of cases of ligament injuries related to sports practice and falls, while the PCL and ACL + PCL groups presented more cases related to traffic accidents and direct trauma caused by an object (weight) $(\mathrm{p}<0.001)$.

Table 1 shows the graph for variables with statistical significance (type of accident). The other variables that were studied did not achieve levels of statistical significance $(\mathrm{p}<0.05)$.

\section{VARUS DEFORMITY GROUP}

Seven patients presented varus deformity of the knee;

Table 1 - Comparison of diagnosis groups with other demographic and clinical variables.

\begin{tabular}{|c|c|c|c|c|c|}
\hline & & \multicolumn{3}{|c|}{ DIAGNOSIS GROUPS } & \multirow[b]{2}{*}{ p value } \\
\hline & & ACL $(n=69)$ & $\mathrm{ACL}+\mathrm{PCL}(\mathrm{n}=17)$ & PCL $(n=23)$ & \\
\hline Age (years) & $\begin{array}{c}\text { Mean } \pm \text { SD } \\
\text { Median } \\
(\text { Min; Max })\end{array}$ & $\begin{array}{c}31.49 \pm 7.67 \\
30 \\
(16 ; 55)\end{array}$ & $\begin{array}{c}29.18 \pm 8.47 \\
28 \\
(20 ; 51)\end{array}$ & $\begin{array}{c}32.26 \pm 7.77 \\
31 \\
(20 ; 47)\end{array}$ & $0.442^{(1)}$ \\
\hline Male gender & & $68(98.6 \%)$ & $17(100.0 \%)$ & $22(95.7 \%)$ & $0.601^{(2)}$ \\
\hline $\begin{array}{l}\text { Time since injury } \\
\text { (months) }\end{array}$ & $\begin{array}{l}\text { Mean } \pm \text { SD } \\
\text { Median } \\
(\text { Min; Max) }\end{array}$ & $\begin{array}{c}30.78 \pm 28.79 \\
24 \\
(3 ; 180)\end{array}$ & $\begin{array}{c}19.00 \pm 14.51 \\
12 \\
(3 ; 48)\end{array}$ & $\begin{array}{c}34.13 \pm 32.69 \\
24 \\
(4 ; 120)\end{array}$ & $0.165^{(3)}$ \\
\hline Right side of the body & & $39(56.5 \%)$ & $12(70.6 \%)$ & $11(47.8 \%)$ & $0.354^{(4)}$ \\
\hline Varus deformity & & $6(8.7 \%)$ & $1(5.9 \%)$ & $0(0.0 \%)$ & $0.449^{(2)}$ \\
\hline Neoligament injury & & $8(11.6 \%)$ & $1(5.9 \%)$ & $0(0.0 \%)$ & $0.204^{(2)}$ \\
\hline $\begin{array}{l}\text { Type of } \\
\text { Accident }\end{array}$ & $\begin{array}{l}\text { Sports } \\
\text { Weight } \\
\text { Fall } \\
\text { Traffic }\end{array}$ & $\begin{array}{c}46(66.7 \%) \\
1(1.5 \%) \\
14(20.3 \%) \\
8(11.6 \%)\end{array}$ & $\begin{array}{c}5(29.4 \%) \\
1(5.9 \%) \\
2(11.8 \%) \\
9(52.9 \%)\end{array}$ & $\begin{array}{c}6(26.1 \%) \\
1(4.4 \%) \\
2(8.7 \%) \\
14(60.9 \%)\end{array}$ & $<0.001^{(2)}$ \\
\hline
\end{tabular}

(1) Descriptive probability level of the Variance Analysis to a factor; (2) Descriptive probability level of Fisher's exact test; (3) Descriptive probability level of Kruskal-Wallis non-parametric test; (4) Descriptive probability level of the chi square test 
of these, two (28.6\%) developed this deformity following an ACL re-injury. The mean age of these patients was 32.14 \pm 9.75 years, all were males and $85.7 \%$ of them had their right side affected. In $71.4 \%$ of patients, the previous ligament injuries had occurred during sports practice.

The varus group showed significantly higher values for the time elapsed between ligament injury and specialized medical treatment $(\mathrm{p}<0.01)$ (Figure 1).

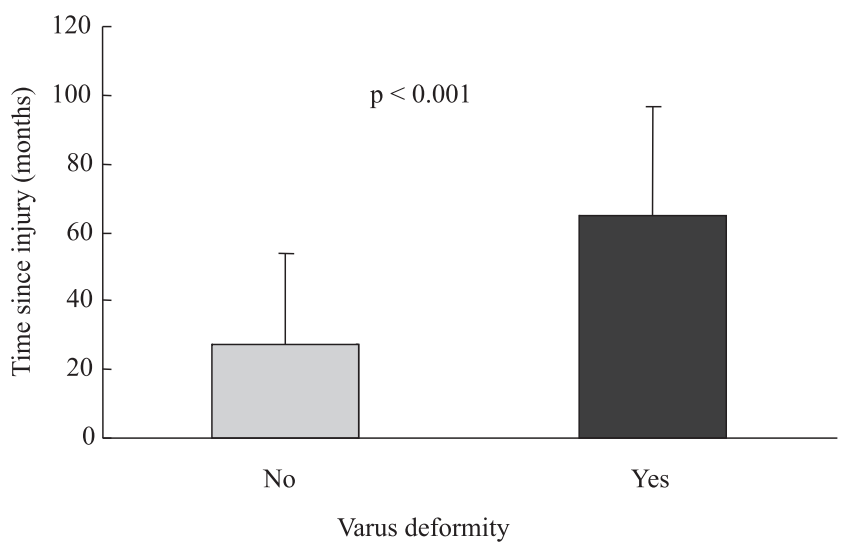

Figure 1 - Graph showing the time since injury for groups with and without varus deformity.

\section{NEOLIGAMENT INJURY GROUP}

Of 109 patients, nine presented with injury of a newly reconstructed ligament (ACL) associated with other ligament injuries in the same joint.

All patients were males, and their mean age was 33.44 \pm 4.5 years. The most affected side was the left side $(55.6 \%)$. Eight of nine patients who suffered injuries of the new ligament acquired the injury during sports practice.

The group with a new ACL injury (neoligament) associated with other ligament injuries had disruption times higher than the group with ACL primary injuries, and this difference was statistically significant $(\mathrm{p}<0.001)$ (Figure 2$)$.

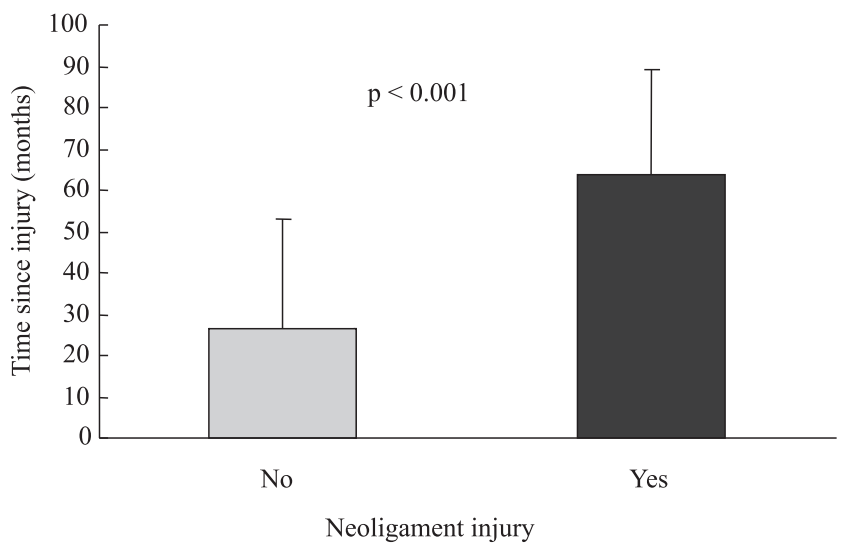

Figure 2 - Graph showing the time since injury for groups with and without neoligament disruption.

\section{DISCUSSION}

Fortunately, multiple ligament injuries of the knee are not frequent ${ }^{2}$. These complex injuries ${ }^{1,2}$ can induce severe sequels if not properly treated.

The majority of knee ligament reconstructions at our institution were performed in young, male sports amateurs. With combined ligament lesions, usually of the right knee, they delayed seeking special medical care for long periods. This delay with regard to surgical treatment may be explained by voluntary postponement by the patient or by difficulty in diagnosing these conditions by previously consulted orthopedic surgeons, who were generally not knee injury specialists.

In this study, the injuries caused by sports practice exceeded those caused by traffic accidents, which corroborates some articles ${ }^{8-10}$. On the other hand, some authors ${ }^{1,2,11}$ quote traffic accidents in their articles as being the most frequent cause, followed by sports accidents. In our "sports injuries" case series soccer practice generated most occurrences of multiple ligament disruptions in the knee. This fact is explained by the widespread participation in this sport in our country.

The group with ACL injury and other combined ligament injuries was more affected during sports practice and accidents with falls in general. The groups involving PCL injuries with other associated ligament disruptions were related to traffic accidents and direct trauma caused by an object (weight). Some authors ${ }^{1,3}$ have drawn attention to the importance of distinguishing between injuries generated by low energy and high energy situations. They have stated that low energy situations are usually associated with sports injuries, while high energy situations are associated with car accidents, as observed in our study.

Combined injuries to the ACL and medial structures are the most frequent complex ligament injuries of the knee ${ }^{12}$. However surgical treatment of the medial area is required only in rare cases ${ }^{13}$. This explains the small number of such cases operated on in this case series.

In this study, among the operated knees, ACL injuries associated with Posterolateral Corner (PLC) injuries were the most frequent multiple ligament tears, both in primary injuries $(n=45)$, in accordance with some studies ${ }^{2,11}$, as well as in ACL reconstructions (neoligament, $\mathrm{n}=6$ ).

Patients affected by injury of the neoligament (ACL) associated with other ligament injuries waited much longer to seek medical advice, with a significant statistical difference. However, they did not develop varus deformities of the knee in a statistically significant manner, as occurred with primary injuries.

Chronic combined ligament disruptions may generate 
knee deformities. ${ }^{6}$ In our study, we observed a directly proportional relationship between angular varus deformity and time since injury that was statistically significant.

\section{CONCLUSIONS}

In our retrospective analysis:

The majority of ACL lesions associated with additional ligament injuries other than PCL injury are related to sports practice and falls.
PCL injury associated with other ligament injuries, regardless of the ACL, is related to traffic accidents and direct trauma caused by an object to the knee (weight).

A significant delay between primary ligament injuries and their reconstructions generates varus deformity of the affected knee.

In spite of their large delays in seeking medical treatment, few patients with injuries of the neoligament (ACL) with other combined disruptions will develop varus deformity.

\section{REFERENCES}

1. Helgeson MD, Lehman Jr. RA, Murphy KP. Initial evaluation of the acute and chronic multiple ligament injured knee. Am J Knee Surg. 2005;18:213-9.

2. Tzurbakis M, Diamantopoulos A, Xenakis T, Georgoulis A. Surgical treatment of multiple knee ligament injuries in 44 patients:2-8 years follow-up results. Knee Surg Sports Traumatol Arthosc. 2006;14:73949.

3. Fanelli GC, Orcutt DR, Edson CJ. The multiple-ligament injured knee:evaluation, treatment and results. Arthoscopy. 2005;21:471-86.

4. Hertel P, Lais E, Bernard M. Optimal treatment of complex ligament injuries and biomachanical aspects. Zentralbl Chir. 1989;114:1489-500.
5. Fanelli GC, Edson CJ, Maish DR. Combined anterior/posterior/medial/ lateral side injuries of the knee. Sports Med Arthrosc Rev. 2001;9:20818.

6. Kawano CT, Bispo Jr. RZ, Oliveira MG, Soejima AT, Apostolopoulos SB. Posterolateral knee instability:an alternative proposal for surgical treatment. Clinics. 2007;62:371-74.

7. Rosner B. Fundamentals of Biostatistics - Boston, PWS Publishers, Second edition, 1986, p. 584.

8. Fanelli GC. Evaluation and treatment of the multiple ligament injured knee. Arthroscopy. 2003;19:30-7.

9. Fanelli GC. PCL injuries in trauma patients. Arthroscopy. 1993;9:2914. 
10. Fanelli GC, Edson CJ. PCL injuries in trauma patients. Part II. Arthroscopy. 1995;11:526-9.

11. Fanelli GC, Edson CJ. Arthoscopically assisted combined anterior and posterior cruciate ligament reconstruction in the multiple ligament injured knee: 2 to10 - years follow-up. Arthoscopy. 2002;18:703-14.
12. Noyes FR, Barber-Westin SD. The treatment of acute combined ruptures of the anterior cruciate and medial ligaments of the knee. Am J Sports Med. 1995;23:380-9.

13. Kim SJ, Choi NH, Shin SJ. Semitendinosus tenodesis for medial instability of the knee. Arthroscopy. 2001;17:660-3. 